Meta

Journal des traducteurs

Translators' Journal

\title{
La grammaire du traducteur
}

\section{Irma Biojout de Azar et Beatriz Amanda Chiappa}

Volume 35, numéro 3, septembre 1990

La traduction dans le monde hispanolusophone

URI : https://id.erudit.org/iderudit/002492ar

DOI : https://doi.org/10.7202/002492ar

Aller au sommaire du numéro

Éditeur(s)

Les Presses de l'Université de Montréal

ISSN

0026-0452 (imprimé)

1492-1421 (numérique)

Découvrir la revue

Citer cet article

Biojout de Azar, I. \& Chiappa, B. A. (1990). La grammaire du traducteur. Meta, 35(3), 499-509. https://doi.org/10.7202/002492ar d'utilisation que vous pouvez consulter en ligne.

https://apropos.erudit.org/fr/usagers/politique-dutilisation/ 


\title{
LA GRAMMAIRE DU TRADUCTEUR
}

\author{
Irma Biojout de AZAR ET BEATrIz AMANDA ChIAPPA \\ Universidad Nacional de La Plata, Argentine
}

\begin{abstract}
Admirable métier celui du traducteur qui connaît parfaitement les deux langues, qui n'ignore la grammaire ni ne se presse pour finir une tâche créatrice qui nous met en rapport intime avec l'auteur traduit et choisi ${ }^{1}$.
\end{abstract}

Le grand dessein du traducteur est de chercher patiemment la façon de jeter un pont entre deux dimensions socio-culturelles et deux systèmes linguistiques souvent très différents. Cet écart cependant n'est pas trop marqué dans notre cas particulier puisque nous mettons en rapport deux langues latines, l'espagnol et le français.

Deux problèmes méthodologiques se posent dans la formation du traducteur: le problème de la compréhension dans la langue de départ et un problème d'expression dans la langue d'arrivée. Nous allons nous occuper dans cet article des grammaires et de leur intérêt pour le traducteur pour résoudre les problèmes d'expression dans la langue d'arrivée.

Silvina Bullrich, dans un compte rendu du roman de Marguerite Duras, Le Viceconsul, souligne l'importance de la grammaire: «Dans le cas du roman que nous commentons aujourd'hui, la différence entre le français et l'espagnol se manifeste surtout à travers la répétition du mot ella, qui devait être supprimé quatre-vingts pour cent des fois. En français, il est impossible de démontrer qui mange, qui dort, qui marche, sans mettre devant le verbe «il» ou «elle». En espagnol, il n'est pas nécessaire de dire ella duerme, ella se despierta, ella tiene hambre. Cela ralentit la lecture. N'est-ce pas déjà une leçon de grammaire?

Nous présentons un bref aperçu de quelques optiques grammaticales illustrées par des exemples tirés de la même revue, publiée en français, La Recherche, et en espagnol, sous le titre Mundo científico. Nous essaierons de montrer à travers eux dans quelle mesure ces descriptions grammaticales sont utiles au traducteur. Nous ne suivons pas un ordre chronologique selon l'ordre d'apparition dans le firmament linguistique de tel ou tel courant, ni une hiérarchisation de telle ou telle école linguistique.

Nous distinguons trois domaines autonomes au niveau de l'analyse, chacun avec son propre fonctionnement et souvent avec des structurations opposées :

1. les grammaires phrastiques (morphosyntaxiques)

1.1. la grammaire générative transformationnelle

1.2. la grammaire systématique

1.3. la grammaire normative

\section{2. les grammaires sémantiques}

2.1. la grammaire notionnelle

2.2. la grammaire des cas

\section{3. les grammaires textuelles}




\section{1. les grammaires phrasiques (morphosyntaxiques)}

\section{1 la grammaire générative transformationnelle}

La grammaire générative transformationnelle pose que les phrases d'une langue ont une double structure: une structure profonde et une structure superficielle. La structure profonde n'apparaît pas à la perception de l'auditeur ou du lecteur, mais elle porte l'interprétation sémantique de la phrase. Après des opérations linguistiques variées, appelées transformations, cette structure profonde se réalise en une structure superficielle. Les transformations portent généralement le nom de l'effet qu'elles produisent sur les phrases noyaux. Ainsi la transformation affixale est une permutation qui affecte les affixes de l'auxiliaire et du nombre; la passivation est l'opération qui transforme une phrase active en une phrase passive; la transformation complétive permet d'enchâsser une phrase noyau $(\mathrm{P})$ dans une autre $(\mathrm{P})$, dite phrase matrice; et ainsi de suite.

Un exemple réussi d'application de cette méthode d'extrapolation est fourni par le développement du chlore par oxydation catalytique ${ }^{2}$.

Un ejemplo de la aplicación de este método de extrapolación lo proporciona el desarrollo del procedimiento de producción del cloro por oxidación catalítica ${ }^{3}$.

Nous constatons dans ces exemples que le français fait subir à la (P) «Le développement (...) fournit un exemple», la transformation passive, qui lui permet d'emphatiser le $\mathrm{SN}^{2}$, le déplaçant en tête de la phrase. La passivation est d'une extrême fréquence en français dans la langue scientifique et technique, tandis que l'espagnol en fait un usage plus restreint, étant donné qu'il a d'autres procédés qui lui permettent de conserver la phrase active, moins coûteuse, tout en déplacant le $\mathrm{SN}^{2}$ en tête de phrase à l'aide du pronom de reprise $l o$.

L'accident était probablement $d \hat{u}$ à une erreur dans le dessin de la tuyauterie reliant deux réacteurs ${ }^{4}$.

El accidente se debió probablemente a un error en el diseño de las tuberías que enlazaban dos reactores 5 .

Étant donné que les verbes devoir, pouvoir et vouloir ne peuvent pas être passivés et que à une erreur n'est pas un complément d'agent, on pourrait expliquer cette phrase réalisée à partir de la $(\mathrm{P})$ théorique :

Une erreur a causé l'accident.

Par des transformations différentes, cette phrase aurait une réalisation en structure superficielle au moyen d'une structure analogue à celle que nous venons de décrire, tandis qu'en espagnol elle se réalise par ce qu'on appelle la pasiva refleja, c'est-à-dire la forme pronominale du verbe, dont parle Dubois dans sa Grammaire structurale. Nous mentionnons à l'appui de cette hypothèse un cas semblable de (P) théorique : l's symbole du pluriel, utilisé dans les règles, ne se réalise pas nécessairement en structure de surface par $\mathbf{s}$, mais par $\mathbf{x}$ ou aux dans bien des cas.

Quant à «reliant», traduit par que enlazaban, la phrase qui se trouve en structure profonde de ces deux constructions superficielles, syntaxiquement différentes, est la même :

La tuyauterie reliait deux réacteurs.

Las tuberías enlazaban dos reactores.

Ces phrases deviennent, par transformation relative:

qui reliait deux réacteurs

que enlazaban dos reactores 
C'est l'étape qu'a conservée l'espagnol, qui est cependant ambiguë à cause des deux pluriels. Le français a appliqué une autre transformation postérieure, la transformation participiale, qui a donné par effacement de «qui» et adjonction de l'affixe «-ant»:

reliant deux réacteurs

(...) on constate que plusieurs cultures ont connu cet art. On en trouve la trace (...)

(...) este arte ha hallado buena acogida en numerosas culturas. En la antigüedad damos con sus huellas en Egipto (...) ${ }^{7}$

Le pronom en est issu des transformations de pronominalisation et de déplacement qu'a subies le $\mathrm{SP}$ de la $(\mathrm{P})$, précédé de de :

On trouve la place de cet art.

En espagnol le pronom «en» n'existe pas, mais il applique à «de cet art» une transformation adjectivale qui donne $\langle s u »$.

Dans tous ces cas (et bien d'autres), le traducteur est arrivé en espagnol à des structures de surface différentes par l'application de transformations autres que celles qu'utilise le français. Il est évident que sa compétence linguistique lui a fait suivre un cheminement différent, sans qu'il ait eu probablement conscience des opérations mentales qu'il a mises en œuvre.

\subsection{La grammaire systématique}

Il est admis sans conteste que la pratique d'une langue est le résultat d'une activité mentale. La science crée par Guillaume part de l'hypothèse suivante: «Si l'acte humain du langage recouvre une activité pensante quelconque, il est forcé que les opérations de pensée impliquées dans cette activité s'accompagnent d'un écoulement minimal de temps».

Même si ce temps est extrêmement bref, il existe réellement. Guillaume expliquait dans ses cours d'une façon familière mais concrète ce principe: «Il faut du temps pour penser comme il faut du temps pour marcher.»

Nous représenterons d'une façon très élémentaire dans le schéma ci-dessous l'incidence du temps dans la catégorie du discours qui s'y prête mieux, c'est-à-dire le verbe. Comme d'autres catégories, les modes verbaux sont des étapes dans l'actualisation de l'image-temps. Au premier moment, la chronogenèse, opération qui permet à la conscience de construire l'image-temps, n'a pas commencé à opérer mais elle est en puissance de le faire. Ce moment correspond à l'infinitif et au participe, qui ne distinguent aucune époque. Vient ensuite un moment intermédiaire où la chronogenèse est en train d'opérer. À ce moment correspond le mode subjonctif, qui distingue mal les époques. Quand la chronogenèse a fini d'opérer, la pensée a réalisé l'image-temps parfaite. C'est le mode indicatif, qui est capable d'exprimer avec netteté les époques différentes.

$\begin{array}{lll}\text { Premier moment } & \text { Moment intermédiaire } & \text { Moment final } \\ \text { Époque }=\text { zéro } & \text { Époque }=\text { ? } & \text { Époque }:+ \\ \text { Personne }=\text { zéro } & \text { Personne }:+ & \text { Personne }:+ \\ \text { (Infinitif) } & \text { (Subjonctif) } & \text { (Indicatif) }\end{array}$

C'est cette référence au temps qui explique la fluctuation entre le subjonctif (époque $=$ ?) et l'infinitif (époque $=$ zéro) dans certaines subordonnées temporelles avec le même sujet de la proposition principale, et le choix que peut éventuellement en faire le traducteur:

Avant que je ne renoue avec lui, j'exige qu'il me présente des excuses.

Avant de renouer avec lui, j'exige qu'il me présente des excuses ${ }^{8}$. 
En espagnol on préfère: «Antes de reconciliarme...»

Avec l'articulateur après que, les deux constructions sont valables aussi, malgré l'indicatif de la subordonnée:

Après qu'il ent quitté Paris, Cézanne retourna en Provence, où il peignit nombre de chefsd'ouvre.

Après avoir quitté Paris ${ }^{9} \ldots$

C'est à cause de sa valeur temporelle zéro que l'infinitif est utilisé à la place d'un subjonctif et même d'un indicatif et qu'on laisse aux prépositions avant ou après le rôle de marquer l'antériorité ou la postériorité.

\subsection{La grammaire normative}

Nous constatons que, sous des optiques différentes ou avec des explications plus poussées et rigoureuses, toutes les grammaires impliquent une norme. La grammaire normative, elle, se borne à enregistrer le «bon usage», celui «des personnes qui ont souci de bien parler et de bien écrire»; elle est définie comme l'art de parler et d'écrire correctement (Grevisse), et sa tâche est de décrire cet usage, cette norme.

L'appareil cardiovasculaire de l'astronaute continue à travailler dans des conditions anormales 10 .

El aparato cardiovascular del astronauta continúa trabajando en condiciones anormales ${ }^{11}$. espagnol.

La rection $\grave{a}+$ infinitif du verbe «continuer», correspond à continuar + gérondif en

En effet, le changement de position corporelle (...) ne peuvent engendrer les phénomènes réflexes qui permettent d'éviter, au sol, l'accumulation du sang dans les parties inférieures du corps ${ }^{12}$.

En efecto, el cambio de posición corporal (...) no pueden engendrar los fenómenos reflejos que permiten evitar, en tierra, la acumulación de la sangre en las partes inferiores del cuerpo ${ }^{13}$.

La rection permettre + de + infinitif devient en espagnol permitir + infinitivo.

Le respect du week-end (sous-titre) $^{14}$

\section{El respeto del fin de semana (subtitulo) 15}

Bien que l'utilisation de l'article défini dans les titres soit facultative en français, on le trouve dans des cas où, en général, l'espagnol ne l'utiliserait pas.

$\mathrm{Ni}$ laxiste, ni fixiste, une bonne grammaire «normative» doit se tenir au juste milieu, en adaptant la norme à la vie moderne, car, de même qu'un purisme exagéré figerait la langue et l'empêcherait de croître et de vivre, l'acceptation de toutes les licences aboutirait à une dégradation.

\section{Les grammaires sémantiques}

\subsection{La grammaire notionnelle}

C'est à travers les structures possibles de la langue d'arrivée que le traducteur rendra le plus grand nombre de nuances possible de la langue de départ. La grammaire notionnelle, organisée à partir de notions de base (hypothèse, opposition, cause, caractérisation, etc.) au lieu de catégories grammaticales, se place du point de vue du locuteur qui veut s'exprimer. Elle lui montre des voies pour choisir ce qui convient au génie de la langue, à la structure, au rythme et même à la musicalité de la phrase: une proposition 
relative, un adjectif qualificatif, un syntagme prépositionnel, un nom, un participe présent, etc.

Bien que ces pertes calciques diminuent avec la pratique d'exercices physiques $(\ldots)^{16}$

Aunque estas pérdidas de calcio disminuyen con la práctica de ejercicios físicos (... $)^{17}$

La caractérisation peut être exprimée de plusieurs manières. Ici, un adjectif qualificatif, «calciques», apparaît traduit par un SP formé par la préposition de + nom.

S'ils [les facteurs dynamiques de vol] posent de sérieux problèmes pour le plan des équipements mécaniques, leur influence sur le plan biologique et médical est relativement peu importante ${ }^{18}$

Aunque estos planteen serios problemas a nivel de los equipos mecánicos y electrónicos, su influencia desde el punto de vista biologico y médico es relativamente poco importante ${ }^{19}$

Parmi les sens possibles de si, le contexte marque l'opposition.

Dans l'espace, l'absence de pesanteur provoque des troubles divers. Mais l'organisme humain s'adapte rapidement 20 .

En el espacio, la ausencia de gravedad provoca diversos problemas, pese a lo cual el organismo humano se adapta rápidamente.

«Mais» correspond à une vaste gamme d'expressions de l'opposition. Ici, pour des raisons de style, le traducteur a choisi une lexie, «pese a lo cual».

\subsection{La grammaire des cas}

Les grammaires sémantiques réfutent la théorie standard de Chomsky. Ainsi, Ch. Fillmore soutient que la forme n'est pas indépendante du sens et que, par conséquent, la structure profonde des phrases est sémantique et se manifeste dans la communication linguistique. Cette conception de la structure profonde permet de donner la même représentation à :

J'aime sa robe. Sa robe me plaît.

L'espagnol rend compte de ces deux phrases par une structure unique:

Me gusta su vestido.

Cette «grammaire des cas» répète le schéma «prédicat-arguments», provenant de la logique, mais chez elle, les arguments sont des cas : agent, instrument, datif, objet, locatif, etc. Dans une phrase telle que :

1. Paul envoie une lettre. $\quad$ 2. Paul reçoit une lettre.

il y a une relation logique entre un prédicat (envoie, reçoit) et des arguments (Paul, lettre). Fillmore remplace ce schéma par le schéma prédicat (cas, cas). Mais la relation sémantique en est différente: dans 1. la relation est agent/objet, dans 2 . datif/objet.

Les cas peuvent se cumuler. Ainsi, dans cette phrase, trois cas accompagnent le procès :

Jean prend la corde avec ses dents.

Agent Procès Objet Instrument phrase :

Mais un même cas ne peut apparaître deux fois dans la structure profonde d'une 
$\begin{array}{llll}\text { Une balle } & \text { a brisé } & \text { la devanture } & \text { avec un fusil. } \\ \text { Instrument } & \text { Procès } & \text { Objet } & \text { Instrument }\end{array}$

(Fillmore)

Certain cas, réalisés comme des phrases, ont la propriété de récursivité:

Paul montre que ses amis pensent qu'il croit qu'ils aimeraient qu'il leur dise qu'ils ont raison.

Certaines distinctions sémantiques peuvent être rendues explicites grâce à la «grammaire des cas». La phrase: «Dominique a fait peur à Ambroise en criant» (donnée par Baylon-Fabre) peut avoir deux interprétations : «Dominique» est l'agent de «fait peur»et de «en criant», ou bien «Dominique» est l'instrument de «fait peur» qui, par conséquent, n'a pas d'agent.

Le lexique permet de déterminer les cas avec lesquels un prédicat peut ou doit être associé, ainsi que les présuppositions et les transformations qui les caractérisent. Fillmore propose l'exemple poursuivre + agent + objet + (instrument $)$ :

$\begin{array}{llll}\text { Jean } & \text { poursuit } & \text { le voleur } & \text { en voiture. } \\ \text { Agent } & \text { Procès } & \text { Objet } & \text { Instrument }\end{array}$

où agent et objet sont obligatoires, et intrument facultatif. Les présuppositions sont: agent et objet + instrument, se déplaçant rapidement. Il n'y a pas de transformations particulières dans ce verbe.

Enfin, le choix du sujet et de l'objet grammaticaux et des prépositions est représenté par des transformations dans la structure superficielle. Cette théorie permet de représenter diverses corrélations entre des faits sémantiques (les cas) et des faits grammaticaux (les fonctions). Ainsi, dans la phrase :

L'auto poursuit le voleur.

où l'agent et le datif ne sont pas exprimés, l'instrument devient le sujet grammatical.

(...) les cirques, les music-halls, présentent tous des numéros de jongleurs (... $)^{22}$

(...) los circos, los music-halls, siempre ofrecen nümeros de malabarismo (... $)^{23}$

«Les cirques, les music-halls» constituent le sujet grammatical; la «grammaire des cas», en revanche, les considère comme des locatifs, comme des lieux où quelqu'un (agent non explicite) présente «les jongleurs» (objet).

Diverses objections ont été faites à cette théorie, parmi lesquelles nous ne retenons que la difficulté d'émettre des jugements sémantiques au sujet de certains cas.

Ce système de relations syntaxiques casuelles a une importance fondamentale dans la description d'une langue. On a dressé un répertoire presque universel de formes casuelles, à côté de formes marginales qui n'ont pas atteint une forme autonome dans toutes les langues. De là l'hypothèse que les relations casuelles sont universelles et qu'elles représentent des universaux conceptuels. Autrement dit, les langues élaborent des moyens pour exprimer ces relations, le cas préexistant au développement de la marque casuelle qui le réalise. Une analyse contrastive pourrait déterminer les correspondances «relation-forme» universelles et les correspondances propres à une langue particulière.

\section{Les grammaires textuelles}

Il est entendu que l'information se répartit dans l'énoncé à travers des structures de langue syntaxiques, sémantiques et phonétiques. Ni les éléments de base de la structure 
formelle (syntaxique), ni le sens d'une phrase ne coïncident forcément avec l'information qu'elle véhicule. Certains segments de l'énoncé apportent plus d'information que d'autres, ce qui nous conduit à nous demander comment ces structures de langue fonctionnent dans l'acte de communication, comment elles sont liées aux structures textuelles, de quelle façon le texte avance avec un apport nouveau d'information, de quelle façon l'information se trouve distribuée dans l'énoncé.

La fonction primaire du langage est la fonction communicative ou référentielle, dont le but est d'apporter une information nouvelle au récepteur. Les éléments de base de cette structure porteuse d'information sont le thème et le rhème; étant donné que tous les éléments de la phrase contribuent plus ou moins au développement de la communication, l'information se distribue selon une échelle, dans laquelle le thème est l'élément qui apporte le moins d'information, «qui porte le degré le plus bas de dynamisme communicatif», et le rhème en a le plus haut (Combettes). Ces notions permettent d'analyser des phrases où tous les éléments sont nouveaux, ayant une partie thématique, moins importante, une transition, qui regroupe tout ce qui ne peut pas être rattaché au thème ou au rhème, enfin le rhème, l'élément le plus important du point de vue de l'apport d'information.

Thème et rhème ne coïncident pas toujours exactement avec les constituants syntaxiques (niveau 1) ou sémantiques (niveau 2). Dans:

Paul envoie une lettre / Paul reçoit une lettre

I au niveau syntaxique les deux phrases ont la même structure: $\mathrm{SN}_{1}+\mathrm{V}+\mathrm{SN}_{2}$;

II au niveau sémantique, Paul est agent dans le premier exemple, datif dans le deuxième;

In au niveau thématique, Paul est dans les deux cas le thème, et le prédicat «envoie une lettre», «reçoit une lettre», le rhème.

Parfois, seule une partie du constituant est concernée :

(Qu'écoute ton frère?) Il écoute/de la musique

Thème Rhème

face à :

(Que fait ton frère?) Il /

Thème Rhème

(Combettes)

D'autres fois, aucun élément de la phrase ne joue la fonction de thème : l'ensemble de la phrase est rhématique, car toute l'information est nouvelle:

(Qu'est-ce qu'il y a ?) Une dame s'est évanouie

$$
\text { Rhème }
$$

La quantité de dioxyde de carbone dans l'atmosphère a augmenté d'environ 290 parties par million (ppm) jusqu'à $320 \mathrm{ppm}$ au cours du siècle dernier ${ }^{24}$. / Rhème

La cantidad de anhidrido carbónico existente en la atmósfera aumentó desde aproximadamente 290 partes por millón (ppm) hasta 320 ppm durante el transcurso del siglo pasa$d o^{25}$. / Rhème

Les moyens mis en œuvre pour que l'information progresse relèvent de quatre domaines différents, chacun avec des réalisations diverses: la situation, l'ordre des syntagmes, les indices sémantiques et l'intonation. 
La situation (ou contexte extra-linguistique), ajoutée au contexte linguistique, peut déterminer la distribution des éléments informationnels dans la phrase. Cf. ci-dessus les exemples avec «Il écoute».

L'ordre des éléments dans la phrase est fondamental dans la distribution de l'information. On tend à placer le thème avant le rhème :

(T) Cette grandeur, sans dimension, $(R)$ représente la quantité relative de flux d'énergie soustraite au rayonnement solaire incident ${ }^{26}$.

Este valor, sin dimensión, representa la cantidad relativa de flujo de energía sustraída a la radiación solar incidente 27.

Cependant, l'ordre inverse peut apparaitre dans des cas particuliers:

(R) Trois chronologies distinctes ont été extrapolées/pour ( $T$ ) vérifier cette hypothèse 28 .

Se ha extrapolado tres cronologias diferentes para verificar esta hipótesis 29 .

Combettes donne les exemples:

Il s'enferme / pour étudier

Thème Rhème

(= but; apport

d'information

nouvelle)

\author{
Pour étudier / il s'enferme. \\ Thème Rhème \\ (= conséquence; \\ apport d'infor- \\ mation nouvelle)
}

Certains indices sémantiques donnent une valeur thématique ou rhématique aux syntagmes où ils se trouvent. Ainsi le déterminant défini, surtout le démonstratif et le possessif, thématisent le syntagme, ainsi que les pronoms personnels; les déterminants indéfinis et des mots tels que «même», «seulement», ont une valeur rhématique; le gallicisme «C'est ... qui / que» rhématise le mot ou le groupe encadré; d'une façon générale le défini se trouve à la gauche de l'indéfini. Cf. les exemples (26) et (27).

La méthode consiste à construire un petit réacteur pilote ${ }^{30}$.

El método consiste en construir un pequeño reactor «piloto» 31 .

Les procédés intonatifs s'adaptent automatiquement au contexte, surtout quand aucun procédé typographique ou sémantique n'apparaît, permettant ainsi de rhématiser des syntagmes qui normalement seraient des thèmes.

Enfin, cette analyse informationnelle peut coïncider avec les analyses syntaxiques et sémantiques:

La pénétration de la tête du spermatozoïde (...) entraîne une cascade de phénomènes 32 .

La penetración de la cabeza del espermatozoide (...) entraña una serie de fenómenos ${ }^{33}$.

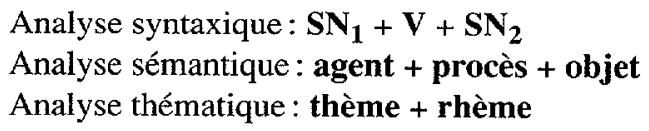

Mais le plus souvent ces coïncidences disparaissent, car aussi bien le thème que le rhème peuvent recouvrir des fonctions syntaxiques différentes.

(T) En attendant, nous continuons / à nous entraîner et à $(\mathrm{R})$ perfectionner nos tours de mains 34 . 
Mientras tanto, seguiremos entrenandonos y perfeccionando nuestros numeros de habili$\mathrm{dad}^{35}$.

(T) Pour le généticien, cela/veut simplement dire que/le (P) programme du développement embryonnaire se met en marche $(R)$ quand les patrimoines génétiques mâle et femelle sont rassemblés 36

Para el genetista, ello quiere decir simplemente que el programa del desarrollo embrionario se pone en marcha cuando los patrimonios genéticos masculinos y femeninos se unen ${ }^{37}$.

Ces phénomènes textuels sont d'une très grande importance pour le traducteur, car ils lui permettent non seulement d'élargir ses connaissances linguistiques, mais de mieux interpréter l'information véhiculée par le texte.

\section{CONCLUSION}

La grammaire est une science qui a à la fois un objet propre (les relations abstraites entre les signes) et une théorie et une méthode qui, appliquées à une pratique spécifique, transforment son objet.

L'essentiel pour le traducteur n'est pas de décrire la grammaire étrangère, mais de l'intérioriser à partir d'hypothèses sur la nature et le fonctionnement des opérations mentales qui sous-tendent les usages qu'on fait de ces formes dans les deux langues mises en rapport, dans notre cas le français et l'espagnol.

Comme vous avez pu le constater à la lecture de nos citations, les exemples sont souvent utilisés pour démontrer la théorie en question. Par conséquent la connaissance d'une théorie grammaticale ne garantit pas l'acquisition de la grammaire. Cependant, même s'il opère (comme pour toute science) une réduction rigoureuse, ce savoir linguistique est indispensable au formateur et au formé, ne serait-ce que pour mieux comprendre sous quelles conditions et par quelles techniques ils sont capables d'appréhender la grammaire étrangère et de la contraster avec la grammaire maternelle.

Une certaine intuition grammaticale (ou sentiment linguistique) est préalable à l'accès à la compréhension ou à l'élaboration d'une description grammaticale, à plus forte raison s'il s'agit de plusieurs descriptions. L'étudiant-traducteur a déjà acquis un modèle métalinguistique dans sa langue maternelle, ce qui rend plus aisé l'apprentissage méthodique de la grammaire étrangère. Besse et Porquier (p. 178) signalent que «cet apprentissage est en lui-même formateur, en ce qu'il donne une méthode de réflexion sur le langage».

Ceci implique que l'étudiant-traducteur doit avoir une bonne connaissance des deux langues mises en rapport, maternelle et étrangère, et le formateur du traducteur une solide formation linguistique et métalinguistique lui permettant d'adopter éventuellement des démarches métalinguistiques qu'il ne suit pas normalement

Si la grammaire, surtout explicite, est parfois récusée par certains formateurs, c'est que souvent ils en ont une connaissance imprécise ou insuffisante. Même si elle seule ne peut assurer l'acquisition de la langue, la grammaire peut introduire cependant une correction partielle au système chaotique et arbitraire de la langue, devenant ainsi un mécanisme servant à limiter ce désordre et à le ramener à une structure rationnelle.

Une fois posé que les descriptions grammaticales ne doivent pas constituer une fin en elles-mêmes, mais la base pour «re-construire» la langue étrangère, la question centrale dont doit tenir compte le formateur du traducteur est de bien déterminer l'adaptation et l'apport de ces descriptions au fonctionnement et aux normes de cette langue. Son expérience devra lui indiquer la manière la plus appropriée d'organiser les données que lui fournissent les grammaires et d'en sélectionner les plus utiles en fonction des besoins de 
l'étudiant et des objectifs visés. Ainsi, il devra tendre à se servir des apports variés que lui offrent les diverses approches que nous venons d'énoncer succinctement (et d'autres que nous ne mentionnerons pas dans notre brève présentation), dans un contexte pédagogique défini, bien qu'elles ne rendent compte que de façon partielle ou fragmentaire du fonctionnement linguistique et que souvent elles laissent de côté certains phénomènes non sélectionnés. Quel que soit le modèle choisi, il devra tenir compte de l'acquis de l'étudiant en langue maternelle pour y superposer de nouvelles connaissances en langue étrangère.

Le professeur de grammaire doit être capable d'un éclectisme qui lui laisse le choix de la solution la meilleure aux problèmes de la traduction, lui ouvrant plusieurs chemins possibles. Il doit être capable surtout de transmettre ce savoir aux étudiants de traduction, les formant solidement, dès le début, à plusieurs modèles linguistiques (au moins deux) relativement différents. La grammaire traditionnelle, malgré ses incohérences, est difficile à déraciner, car elle est trop ancrée dans l'enseignement aussi bien de la langue étrangère que de la langue maternelle. L'enseignement des théories modernes est indispensable; parmi les plus récentes et les plus rigoureuses, la grammaire générative transformationnelle est très enrichissante, car elle permet au traducteur d'opérer des transformations substantielles sur les phrases de base et d'en choisir les formes les mieux adaptées à son texte. Les grammaires sémantiques et textuelles fournissent les moyens de mieux en saisir le sens.

Le choix d'une optique grammaticale déterminée ne doit pas exclure une information sur d'autres modèles modernes. Cette formation grammaticale diversifiée a l'avantage d'exercer enseignants et enseignés à la construction rigoureuse d'un système grammatical, et de pouvoir se recycler et suivre l'évolution de cette discipline dans des livres et des revues, grâce à leur initiation à ce métalangage et à leur entraînement intellectuel.

\section{NOTES}

1. L'épigraphe a été tirée de Silvana Bullrich, Despedida a Simone de Beauvoir, in La Nacíon Buenos Aires, $1^{\text {er }}$ avril 1986. (Notre traduction.)

Les exemples ont été tirés de la même tevue publiée en français et en espagnol:

La Recherche, Paris, 1982

Mundo cientifico. La Recherche, versión en castellano, Valence, Fontalba, 1982

Les chiffres renvoient aux citations; les chiffres entre parenthèses, aux pages où se trouvent ces citations.

Les exemples (4) et (5) peuvent recevoir aussi une interprétation sémantique :

Cela est dû̀ à quelque chose.

Esto es debido/se debe a algo.

Ce n'est pas un passif, car il n'y a pas d'actif correspondant, ni opposition de position de $\mathrm{SN}_{1}$ et $\mathrm{SN}_{2}$. C'est une lexie à forme passive exprimant la cause.

2. (873), 4 (875), 30 (870). VILLERMAUX, Jacques, «Les réacteurs chimiques» ( $n^{\circ} 135$ )

3. (979), 5 (980), 31 (975). VILLERMAUX, Jacques, «Los reactores químicos» ( $\left.\mathrm{n}^{\circ} 18\right)$.

6. (857), 22 (858), 34 (867). GRAHAM, R. et BUHLER, J., «L'Art de jongler» ( $\mathrm{n}^{\circ}$ 135)

7. (961), 23 (962), 35 (970). GRAHAM, R. et BUHLER, J., «Los juegos malabares» ( $\left.\mathrm{n}^{\circ} 18\right)$.

10. (596), 12 (596), 14 (600), 16 (594), 18 (594), 20 (599). PLANEL, N., «La biologie en apesanteur» (no 133).

11. (703), 13 (703), 15 (707), 17 (700), 21 (706), 19 (700). PLANEL, N., «La biología en la ingravidez» ( $\left.\mathrm{n}^{\circ} 16\right)$.

24. (853), 26 (851), 28 (851). REID, A. Bryson, «Volcans et climat» (no 135).

25. (957), 27 (955), 29 (956). REID, A. Bryson, «Volcanes y clima» ( $\left.n^{\circ} 18\right)$.

32. (834). KELLY, Françoise, «Les manipulations génétiques des embryons» (nº 135$)$.

33. (934). KELLY, Françoise, «Manipulaciones genéticas en embriones» (n ${ }^{\circ} 18$ ). 
36. (895). «Nouvelles internationales. Les facteurs maternels de l'ceuf de souris» (no 135).

37. (1006). «Informaciones y noticias. Los factores maternos del huevo de ratón» ( $\mathrm{n}^{0} 18$ ).

8 et 9 (56). HINARD, A. et L. IDRAY : À la découverte de notre langue. $4^{\mathrm{e}}$ et $3^{\mathrm{e}}$ Cycle d'orientation, Paris, Magnard, 1981.

\section{BIBLIOGRAPHIE}

BAYLON, Chr. et P. FABRE (1980), Grammaire systématique de la langue française, Paris, Nathan.

BAYLON, Chr. et P. FABRE (1979), la Sémantique, Paris, Nathan.

BENAMOU, M. et J. CARDUNER (1974), le Moulin à paroles, Paris, Hachette.

BESSE, H. et R. PORQUIER (1986), Grammaire et didactique des langues, Paris, Hatier.

COMBETTES, B. (1983), Pour une grammaire textuelle, Bruxelles, A. De Boeck.

DUBOIS, J. (1967), Grammaire structurale du français. Le Verbe, Paris, Larousse.

DUBOIS, J. et F. DUBOIS-CHARLIER (1970), Éléments de linguistique française : syntaxe, Paris, Larousse.

DUBOIS-CHARLIER, F. (1975), «Avant-propos. Les premiers articles de Fillmore», Langages, Paris, DidierLarousse, juin $1975, \mathrm{n}^{\circ} 38$.

FILLMORE, Ch. (1968), «The Case for Case», in E. BACH et R. T. HARMS, Universals in Linguistic Theory, New York, Holt, Rinehart and Winston.

GÉMAR, Jean-Claude (1981), Séminaire sur la traduction. Fait à l'occasion du 2 e Colloque sur l'enseignement fonctionnel du français et de la traduction en Amérique latine, CEFETAL, Buenos Aires.

GREVISSE, Maurice (1980), le Bon usage, Paris-Gembloux, Duculot.

VALIN, R. (1954), Petite Introduction à la psychomécanique du langage, Québec, Presses de l'Université Laval. 